\title{
Quantum model of cooling and force sensing with an optically trapped nanoparticle: supplementary material
}

\author{
B. Rodenburg ${ }^{1,2, *}$, L. P. NeUKIRCH ${ }^{2,3}$, A. N. VAmivaKas ${ }^{2,4}$, And \\ M. BHATTACHARYA ${ }^{1,2}$ \\ ${ }^{1}$ School of Physics and Astronomy, Rochester Institute of Technology, Rochester, NY 14623, USA \\ ${ }^{2}$ Center for Coherence and Quantum Optics, University of Rochester, Rochester, NY 14627, USA \\ ${ }^{3}$ Department of Physics and Astronomy, University of Rochester, Rochester, NY 14627, USA \\ ${ }^{4}$ Institute of Optics, University of Rochester, Rochester, NY 14627, USA \\ *Corresponding author: brandon.rodenburg@gmail.com
}

Published 17 March 2016

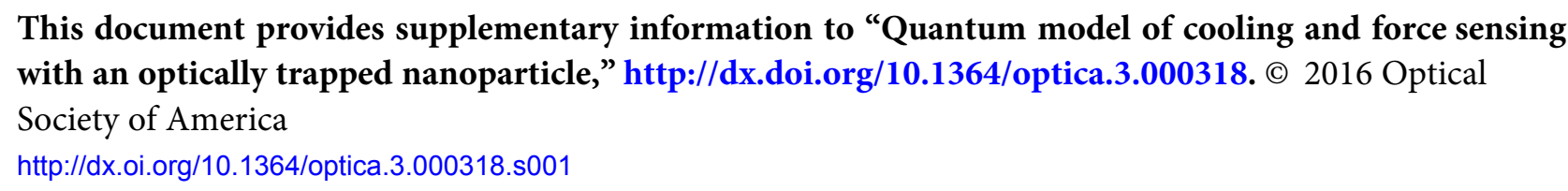

\section{ELECTRIC FIELDS}

The total electric field as defined in the main article is written as

$$
\mathbf{E}(\mathbf{r}, t)=\mathbf{E}_{\mathbf{t}}(\mathbf{r}, t)+\mathbf{E}_{\mathbf{p}}(\mathbf{r}, t)+\mathbf{E}_{\mathbf{b}}(\mathbf{r}, t) .
$$

The $\mathbf{E}_{\mathbf{t}}$ and $\mathbf{E}_{\mathbf{b}}$ fields are both assumed to be Gaussian beams with the trap treated classically and the probe treated as a quantized beam with frequency $\omega_{p}$, linewidth $\Delta \omega$, waist $w_{0}$, and canonical bosonic operators $\left[a, a^{\dagger}\right]=1[1]$

$$
\mathbf{E}_{\mathbf{p}}(\mathbf{r}, t)=i\left(\frac{\hbar \omega_{p} \Delta \omega}{4 \pi \epsilon_{0} c}\right)^{1 / 2} e^{i \omega_{p}(z / c-t)} \mathbf{e}_{\mathbf{p}} G\left(\mathbf{r}^{\prime}, \omega_{p}\right) a+\text { h.c., }
$$

where $G(\mathbf{r}, \omega)$ is the spatial mode function of the beam. The background field $\mathbf{E}_{\mathbf{b}}$ is simply the quantum field of all other modes and can be represented via a typical plane-wave expansion [2].

\section{FREE FIELD HAMILTONIAN}

Our configuration Hamiltonian as given in the main text is

$$
H=H_{m}+H_{f}+H_{\text {int }}
$$

where $H_{m}=p^{2} / 2 m$ is the particle's kinetic energy for the momentum $p=\sqrt{p_{x}^{2}+p_{y}^{2}+p_{z}^{2}}$, and $H_{f}$ and $H_{\text {int }}$ are the free field and interaction Hamiltonians respectively. The energy of the free field Hamiltonian is

$$
H_{f}=\epsilon_{0} \int|\mathbf{E}(\mathbf{r}, \mathbf{t})|^{2} \mathrm{~d}^{3} \mathbf{r} .
$$

The term proportional to $\left|\mathbf{E}_{\mathbf{t}}(\mathbf{r})\right|^{2}$ corresponds to the energy of the trap beam, and can be neglected as it represents a constant offset of the Hamiltonian. The energy of the free probe field is given by [1]

$$
\epsilon_{0} \int\left|\mathbf{E}_{\mathbf{p}}(\mathbf{r})\right|^{2} \mathrm{~d}^{3} \mathbf{r}=\hbar \omega_{p} a^{\dagger} a
$$

The energy of the background field, which can be found in several textbooks (e.g. [2]) can be written in a plane wave expansion as

$$
H_{B}=\sum_{\mu} \int \mathrm{d}^{3} \mathbf{k} \hbar \omega_{\mathbf{k}} a_{\mu}^{\dagger}(\mathbf{k}) a_{\mu}(\mathbf{k}) .
$$

The cross term between the trap and background fields, given by $\int\left(\mathbf{E}_{\mathbf{t}}+\mathbf{E}_{\mathbf{p}}\right) \cdot \mathbf{E}_{\mathbf{b}} \mathrm{d}^{3} \mathbf{r}$, vanishes as their mutual overlap is very small. This cancellation also represents the avoidance of selfinterference and mode overcounting in our model. The cross term between the probe and the background fields vanishes for the same reason. The cross term between the trap and probe fields given by $\mathbf{E}_{\mathbf{t}} \cdot \mathbf{E}_{\mathbf{p}}$, vanishes due to polarization orthogonality. Finally, combining Eqs. (S5) and (S6), the free field Hamiltonian is

$$
H_{f}=\hbar \omega_{p} a^{\dagger} a+\sum_{\mu} \int \mathrm{d}^{3} \mathbf{k} \hbar \omega_{\mathbf{k}} a_{\mu}^{\dagger}(\mathbf{k}) a_{\mu}(\mathbf{k})
$$

which is simply the sum of the probe and background field energies. 


\section{INTERACTION HAMILTONIAN}

The interaction Hamiltonian between the fields and the nanoparticle is given by

$$
H_{\text {int }}=-\frac{1}{2} \int \mathbf{P}(\mathbf{r}) \cdot \mathbf{E}(\mathbf{r}) \mathrm{d}^{3} \mathbf{r} .
$$

Assuming that the particle has a linear polarizability described by $\mathbf{P}(\mathbf{r})=\alpha_{p} \mathbf{E}(\mathbf{r})$, the interaction Hamiltonian is

$$
H_{\text {int }}=-\frac{\epsilon_{0} \epsilon_{\mathcal{C}}}{2} \int_{V}|\mathbf{E}(\mathbf{r})|^{2} \mathrm{~d}^{3} \mathbf{r},
$$

where $\epsilon_{c}=3\left(\epsilon_{r}-1\right) /\left(\epsilon_{r}+2\right)$ is the Clausius-Mossotti relation for the effective relative permittivity of a dielectric due to local field effects, and $V$ denotes integration over the volume of the dielectric particle.

Now when we use the total electric field from Eq. (S1) in the above equation, we again get a number of terms. The terms proportional to $\left|\mathbf{E}_{\mathbf{b}}\right|^{2}$ and $\left|\mathbf{E}_{\mathbf{t}} \cdot \mathbf{E}_{\mathbf{p}}\right|^{2}$ we neglect as these represent a renormalization of the background modes and we again assume the trap and probe are cross-polarized.

\section{A. Trap potential}

The first non-negligible term is the effect of the trap beam proportional to $\int_{V}\left|\mathbf{E}_{\mathbf{p}}\right|^{2} \mathrm{~d}^{3} \mathbf{r}$. Since the nanoparticle is smaller than the wavelength of any relevant optical field, the integration can be written as $\int_{V} \mathrm{~d}^{3} \mathbf{r}=V \int \delta(\mathbf{q}) \mathrm{d}^{3} \mathbf{r}$, where $\mathbf{q}$ is the center of mass position of the particle. Therefore $H_{\text {int }}$ for the trap-particle interaction is given by

$$
\begin{gathered}
-\frac{\epsilon_{c} \epsilon_{0}}{2} V\left|\mathbf{E}_{\mathbf{t}}(\mathbf{q})\right|^{2}=-\frac{\epsilon_{c} \epsilon_{0}}{2} V E_{0}^{2} w_{0}^{2} \frac{\pi}{2}|G(\mathbf{q})|^{2} \\
\approx \frac{\epsilon_{c} \epsilon_{0}}{2} V E_{0}^{2}\left(-1+\left(\frac{q_{z}}{z_{R}}\right)^{2}+2\left(\frac{q_{x}}{w_{0}}\right)^{2}+2\left(\frac{q_{y}}{w_{0}}\right)^{2}\right),
\end{gathered}
$$

where $q_{z}$ is the longitudinal coordinate, and $q_{x}$ and $q_{y}$ are the transverse coordinates. We note that to second order in $\mathbf{q}$, the three degrees of freedom $x, y$, and $z$ decouple, and therefore act as three independent trapping potentials. Ignoring the overall constant in the above equation, we finally have for our trap Hamiltonian

$$
H_{\text {trap }}=\frac{p^{2}}{2 m}+\frac{1}{2} m\left(\omega_{z}^{2} q_{z}^{2}+\omega_{x}^{2} q_{x}^{2}+\omega_{y}^{2} q_{y}^{2}\right)
$$

where $\omega_{z}=\sqrt{\epsilon_{c} \epsilon_{0} E_{0}^{2} V /\left(m z_{R}^{2}\right)}$ and $\omega_{x, y}=\sqrt{2 \epsilon_{c} \epsilon_{0} E_{0}^{2} V /\left(m w_{0}^{2}\right)}$. Writing our canonical nanoparticle variables as

$$
\begin{aligned}
& q_{j}=\sqrt{\hbar /\left(2 m \omega_{j}\right)}\left(b_{j}^{\dagger}+b_{j}\right)=\ell_{j}\left(b_{j}^{\dagger}+b_{j}\right)=\ell_{j} Q_{j} \\
& p_{j}=i \sqrt{\hbar m \omega_{j} / 2}\left(b^{\dagger}-b\right),
\end{aligned}
$$

allows us to rewrite Eq. (S11) as $H_{\text {trap }}=\sum_{j} \hbar \omega_{j} b_{j}^{\dagger} b_{j}$.

\section{B. Optomechanical coupling}

Now we examine the coupling between the probe beam and the trapped nanoparticle given. The Hamiltonian for this term is

$$
\begin{aligned}
H_{\mathrm{OM}}= & -\frac{\epsilon_{c} \epsilon_{0}}{2} \int_{V}\left|\hat{\mathbf{E}}_{\mathbf{p}}(\mathbf{r})\right|^{2} \mathrm{~d}^{3} \mathbf{r} \\
= & -V \frac{\epsilon_{c} \hbar \omega_{p} \Delta \omega}{4 \pi c}\left(a^{\dagger} a+\frac{1}{2}\right)|G(\mathbf{q}-\Delta \mathbf{r})|^{2} \\
= & -V \frac{\epsilon_{c} \hbar \omega_{p} \Delta \omega}{2 \pi^{2} w_{0}^{2} c}\left(a^{\dagger} a+\frac{1}{2}\right) \\
& \times\left(1+2 \frac{\Delta z}{z_{R}^{2}} q_{z}+\frac{4}{w_{0}^{2}}\left(\Delta x q_{x}+\Delta y q_{y}\right)+\mathcal{O}\left(\mathbf{q}^{2}\right)\right),
\end{aligned}
$$

where we have assumed that the probe beam is shifted from the trap beam by a small amount $\Delta \mathbf{r} \equiv(\Delta x, \Delta y, \Delta z)$. Now the terms proportional to a constant times either $a^{\dagger} a$ or $q_{j}$ (for $j \in\{x, y, z\}$ ) can be incorporated into shifts in the optical and mechanical frequencies, and the oscillator position. The optomechanical coupling term between the probe and particle is then given by

$$
\begin{aligned}
H_{\mathrm{OM}}=- & V \frac{\epsilon_{c} \hbar \omega_{p} \Delta \omega}{\pi c} a^{\dagger} a \\
& \times\left(\frac{\Delta z}{z_{R}^{2}} \ell_{z} Q_{z}+2 \frac{\Delta x}{z_{R}^{2}} \ell_{x} Q_{x}+2 \frac{\Delta y}{z_{R}^{2}} \ell_{y} Q_{y}\right) \\
= & -\sum_{j} \hbar g_{j} a^{\dagger} a Q_{j} .
\end{aligned}
$$

The system Hamiltonian is then a combination of the energies of the probe field, optical trap, and optomechanical coupling and is given by

$$
H_{S}=\hbar \omega_{p} a^{\dagger} a+\sum_{j} \hbar \omega_{j} b_{j}^{\dagger} b_{j}-\sum_{j} \hbar g_{j} a^{\dagger} a\left(b_{j}+b_{j}^{\dagger}\right) .
$$

\section{Optical scattering}

Computing the cross coupling Hamiltonian between the field and the background due to scattering by the nanoparticle gives

$$
\begin{aligned}
-\epsilon_{c} \epsilon_{0} \int_{V} \mathbf{E}_{\mathbf{t}} \cdot \mathbf{E}_{\mathbf{b}} \mathrm{d}^{3} \mathbf{r} \approx & -i \frac{\epsilon_{c} V}{2}\left(\frac{\hbar \epsilon_{0}}{16 \pi^{3}}\right)^{1 / 2} \int \mathrm{d}^{3} \mathbf{k} \sqrt{\omega_{k}} \sum_{\mu} \\
& \times\left[1+i\left(\mathbf{k}_{\perp} \cdot \mathbf{q}_{\perp}+\left(k_{z}-k_{0}\right) q_{z}\right)\right] \\
& \times \mathbf{e}_{\mu}(\mathbf{k}) \cdot \mathbf{E}_{0}^{*} a_{\mu}(\mathbf{k}) e^{-i\left(\omega_{k}-\omega_{t}\right) t} \\
& + \text { h.c., }
\end{aligned}
$$

for the trap beam and

$$
\begin{aligned}
-\epsilon_{c} \epsilon_{0} \int_{V} \mathbf{E}_{\mathbf{p}} \cdot \mathbf{E}_{\mathbf{b}} \mathrm{d}^{3} \mathbf{r} & \\
\approx & -i \frac{\hbar \epsilon_{c} V}{8 \pi^{2} w_{0}} \sqrt{\frac{2 \omega_{p} \Delta \omega}{\pi c}} \int \mathrm{d}^{3} \mathbf{k} \sqrt{\omega_{k}} \sum_{\mu} \\
& \times\left[1+i\left(\mathbf{k}_{\perp} \cdot \mathbf{q}_{\perp}+\left(k_{z}-k_{0}\right) q_{z}\right)\right] \\
& \times \mathbf{e}_{p} \cdot \mathbf{e}_{\mu}(\mathbf{k}) a_{\mu}^{\dagger}(\mathbf{k}) a_{p} e^{-i\left(\omega_{p}-\omega_{k}\right) t}+\text { h.c., }
\end{aligned}
$$

for the probe beam, assuming elastic scattering and amplitude of the particle motion small relative to an optical wavelength [3]. Applying standard Born-Markov theory and tracing over the background optical modes and particle motion transverse to the $z$-axis allows us to derive a master equation

$$
\begin{aligned}
\dot{\rho}(t)= & -\frac{7 \omega_{t}^{5}}{\hbar c^{6}} \frac{\left(E_{0}^{2} \epsilon_{0} c / 2\right) \epsilon_{c}^{2} V^{2} \ell_{z}^{2}}{60 \pi} \mathcal{D}\left[Q_{z}\right] \rho(t) \\
& +\frac{1}{\hbar^{4} c^{3}} \frac{\left(\hbar \omega_{p}\right)^{4} \epsilon_{c}^{2} V^{2}}{24 \pi^{3} w_{0}^{2}(c / \Delta \omega)} \\
& \times\left(\mathcal{D}\left[a_{p}\right]+\frac{7 \omega_{p}^{2} \ell_{z}^{2}}{5 c^{2}} \mathcal{D}\left[a q_{z}\right]\right) \rho(t) \\
= & -\frac{A_{t}}{2} \mathcal{D}\left[Q_{z}\right]+\mathcal{L}_{\mathrm{sc}}[\rho(t)]
\end{aligned}
$$


For reference, if we do not trace over the transverse motion, the three-dimensional analogue of the trap scattering is

$$
\begin{aligned}
\mathcal{L}_{t}^{3 D}[\rho(t)] \equiv & -\frac{\omega_{t}^{5}}{\hbar c^{6}} \frac{\left(E_{0}^{2} \epsilon_{0} c / 2\right) \epsilon_{c}^{2} V^{2}}{60 \pi} \\
& \times\left(2 \mathcal{D}\left[q_{\times}\right]+\mathcal{D}\left[q_{\|}\right]+7 \mathcal{D}\left[q_{z}\right]\right) \rho(t),
\end{aligned}
$$

where $\|$ and $\times$ indicate transverse motion parallel (or not parallel) to the polarization direction of the trap beam. A similar expression holds for the probe

$$
\begin{aligned}
\mathcal{L}_{s c}^{3 D}[\rho(t)] \equiv & \frac{1}{\hbar^{4} c^{3}} \frac{\left(\hbar \omega_{p}\right)^{4} \epsilon_{c}^{2} V^{2}}{24 \pi^{3} w_{0}^{2}(c / \Delta \omega)}\left\{\mathcal{D}\left[a_{p}\right]+\frac{\omega_{p}^{2} \ell_{z}^{2}}{5 c^{2}}\right. \\
& \left.\times\left(\mathcal{D}\left[a_{p} q_{\times}\right]+2 \mathcal{D}\left[a_{p} q_{\|}\right]+7 \mathcal{D}\left[a_{p} q_{z}\right]\right)\right\} \rho(t) .
\end{aligned}
$$

To get only the one-dimensional equation for $z$, we can trace over $q_{\times}, q_{\|}$, and $a_{p}$. Since $\operatorname{Tr}_{A}\{\mathcal{D}[A] \rho\} \equiv 0$, we have for the trap

$$
\mathcal{L}_{t}^{1 D}\left[\rho_{z}\right]=-7 \frac{\omega_{t}^{5}}{\hbar c^{6}} \frac{\left(E_{0}^{2} \epsilon_{0} c / 2\right) \epsilon_{c}^{2} V^{2}}{60 \pi} \mathcal{D}\left[q_{z}\right] \rho_{z}
$$

and for the probe

$$
\mathcal{L}_{s c}^{1 D}\left[\rho_{z}\right]=\frac{7}{\hbar^{4} c^{3}} \frac{\left(\hbar \omega_{p}\right)^{4} \epsilon_{c}^{2} V^{2}}{24 \pi^{3} w_{0}^{2}(c / \Delta \omega)} \frac{\omega_{p}^{2} \ell_{z}^{2}}{5 c^{2}}\left\langle a_{p}^{\dagger} a_{p}\right\rangle \mathcal{D}\left[q_{z}\right] \rho_{z} .
$$

\section{DERIVATION OF THE DETECTED HOMODYNE CUR- RENT AND FEEDBACK}

In order to find the input-output relations for the probe field, we write the system Hamiltonian (for the single degree of free$\left.\operatorname{dom} Q_{z}\right)$ as $H_{s}=\hbar \omega_{p} a^{\dagger} a+\hbar \omega_{z} b_{z}^{\dagger} b_{z}-\hbar g a^{\dagger} a Q_{z}$. If we move into the interaction picture for the probe field where $a \rightarrow a e^{-i \omega_{p} t}$, then this becomes $H_{s}=\hbar \omega_{z} b_{z}^{\dagger} b_{z}-\hbar g a^{\dagger} a Q_{z}$.

We assume the probe field is initially a coherent state which can be written as $a=-i \alpha+v$, where $\alpha$ is a constant and $v$ is a field annihilation operator. In this case the optomechanical coupling in our system Hamiltonian becomes

$$
H_{\mathrm{OM}}=-\hbar g_{z} Q_{z} a^{\dagger} a=\hbar g_{z} Q_{z}\left(|\alpha|^{2}+i \alpha v^{\dagger}-i \alpha^{*} v\right) \text {. }
$$

The term proportional to $|\alpha|^{2} Q_{z}$ is responsible for simply shifting the mean position of the oscillator and can safely be ignored, leaving us with $H_{\mathrm{OM}}=i \hbar g_{z} Q_{z}\left(\alpha v^{\dagger}-\alpha^{*} v\right)$.

The Heisenberg equation of motion for $v$ is given by

$$
\dot{v}=\frac{1}{i \hbar}[a, H]=\alpha g_{z} Q_{z},
$$

which can be integrated formally to give

$$
v(t)=v\left(t_{0}\right)+\int_{t_{0}}^{t} \alpha g_{z} Q_{z} \mathrm{~d} t^{\prime} \approx v\left(t_{0}\right)+\alpha g_{z} Q_{z} \Delta t,
$$

where the integration is taken over a time $\Delta t$ short compared to $1 / \omega_{z}$. If we had picked an initial time $t_{f}>t$ then we would have computed $v(t)=v\left(t_{f}\right)-\alpha g_{z} Q_{z} \Delta t$. By identifying the input state as $a_{\mathrm{in}}=-i \alpha+v\left(t_{0}\right)$, and the output state as $a_{\text {out }}=-i \alpha+v\left(t_{f}\right)$, then we can relate the output and input fields by

$$
a_{\mathrm{out}}=a_{\mathrm{in}}+2 \alpha g_{z} \Delta t \equiv a_{\mathrm{in}}+\frac{\alpha \chi}{2} Q_{z}
$$

where we have defined the variable $\chi \equiv 4 g_{z} \Delta t$.

We have a detected homodyne current that is given by

$$
I_{h}=\chi^{2} \Phi\left\langle Q_{z}\right\rangle(t)+\sqrt{\chi^{2} \Phi} \xi(t),
$$

where we have converted from photon numbers to rates by using the identity for the photon flux $\Phi \equiv\left\langle a^{\dagger} a\right\rangle \Delta \omega=\alpha^{2} \Delta \omega$, and have introduced the stochastic variable $\xi(t)$ which is due to the shot noise of the detection.

As described in the main text, we choose to modulate the trap intensity $I_{t} \rightarrow I_{t}(1+M(t))$ such that the modulation depth $M$ is proportional to

$$
\begin{aligned}
M \propto & \frac{I_{h}(t) I_{h}(t-\delta t)}{\chi^{2} \Phi} \\
= & \left(\sqrt{\chi^{2} \Phi}\left\langle Q_{z}(t)\right\rangle+\xi(t)\right) \\
& \times\left(\sqrt{\chi^{2} \Phi}\left\langle Q_{z}(t-\delta t)\right\rangle+\xi(t-\delta t)\right) \\
= & \chi^{2} \Phi\left\langle Q_{z}(t)\right\rangle\left\langle Q_{z}(t-\delta t)\right\rangle+\sqrt{\chi^{2} \Phi}\left\langle Q_{z}(t-\delta t)\right\rangle \xi(t) \\
& +\sqrt{\chi^{2} \Phi}\left\langle Q_{z}(t)\right\rangle \xi(t-\delta t)+\xi(t) \xi(t-\delta t),
\end{aligned}
$$

where the last term is identically zero for $\delta t \neq 0$ as $\xi(t)$ is delta function correlated in time (i.e. $\left\langle\xi\left(t_{1}\right) \xi\left(t_{2}\right\rangle=\delta\left(t_{1}-t_{2}\right)\right.$ ). For this same reason the two noise terms $\left\langle Q_{z}(t-\delta t)\right\rangle \xi(t)$ and $\left\langle Q_{z}(t)\right\rangle \xi(t-\delta t)$ are independent noises with zero mean (due to $\langle\xi\rangle=0$ ), and square given by $2\left\langle Q_{z}\right\rangle^{2}$. Therefore we can combine these terms into a single effective noise term equal to $\sqrt{2 \chi^{2} \Phi}\left\langle Q_{z}\right\rangle \xi(t)$. Assuming the optimal phase is chosen (i.e. $\delta t$ is chosen such that $\left.\left\langle Q_{z}(t-\delta t)\right\rangle=\left\langle P_{z}\right\rangle\right)$, then our final feedback Hamiltonian can be written as

$$
H_{\mathrm{fb}}=\hbar G\left(\chi^{2} \Phi\langle P\rangle+\sqrt{2 \chi^{2} \Phi} \xi(t)\right) Q^{3}=\hbar G I_{\mathrm{fb}} Q^{3},
$$

where we have defined an effective feedback current

$$
I_{\mathrm{fb}} \equiv \chi^{2} \Phi\langle P\rangle+\sqrt{2 \chi^{2} \Phi} \xi(t),
$$

where the factor of two in the second term signifies the additional noise contributed by frequency doubling. Now writing $\sigma \equiv \chi^{2} \Phi P_{z}$ allows us to write the master equation for the feedback in standard notation [4]

$$
\begin{aligned}
\dot{\rho} & =\mathcal{K}\left[\sigma \rho+\rho \sigma^{\dagger}\right]+\frac{2 \chi^{2} \Phi}{2} \mathcal{K}^{2}[\rho] \\
& \equiv-i \chi^{2} \Phi G\left[Q_{z}^{3},\left\{P_{z}, \rho\right\}\right]-\chi^{2} \Phi G^{2} \mathcal{D}\left[Q_{z}^{3}\right] \rho
\end{aligned}
$$

(S31)

where the Liouvillian superoperator $\mathcal{K}$ is defined as $\mathcal{K}[\rho]=$ $[F, \rho] / i \hbar$ and where $F$ is the feedback term that comes from the feedback Hamiltonian $H_{\mathrm{fb}}=I_{\mathrm{fb}} F$, and is chosen to be $F=\hbar G Q_{z}^{3}$ to match the classical physics [5] of the problem as described in the main text. The gain coefficient $G$ may be related to the experimental trap beam intensity modulation $M \equiv \Delta I_{t} / I_{t}$ by using the fact that $H_{\mathrm{fb}}=\hbar \omega_{z} M Q_{z}^{2}=I_{\mathrm{fb}} F$, and therefore [5]

$$
M=\frac{G \chi^{2} \Phi\left\langle Q_{z}\right\rangle\left\langle P_{z}\right\rangle}{\omega_{z}} \approx \frac{G \chi^{2} \Phi N}{\omega_{z}}
$$




\section{FULL MASTER EQUATION}

In the main text of this paper we presented the master equations describing the density matrix of only the single degree of freedom represented by the $z$ motion of the nanoparticle. For convenience, we present the full master equation of the threedimensional motion of the nanoparticle, as well as of the probe beam itself, which can be used to calculate the statistics of any quantum observable for the nanoparticle or probe beam.

The master equation for the density matrix $\rho$ describing the full three-dimensional motion of the nanoparticle, as well as the probe field is given by

$$
\begin{aligned}
\left.\dot{\rho}(t)=\frac{1}{i \hbar}\left[H_{S}, \rho(t)\right]\right\} \text { Unitary dynamics } \\
\left.+\mathcal{L}_{t}^{3 D}[\rho(t)]+\mathcal{L}_{\mathrm{sc}}^{3 D}[\rho(t)]\right\} \text { Light scattering } \\
\left.+\mathcal{B}^{3 D}[\rho(t)]\right\} \text { Gas scattering, }
\end{aligned}
$$

where

$$
\begin{aligned}
\mathcal{B}^{3 D}[\rho]= & -\frac{\eta_{f} k_{B} T}{\hbar^{2}}[\mathbf{q},[\mathbf{q}, \rho(t)]] \\
& -\frac{\eta_{f}}{12 k_{B} T m^{2}}[\mathbf{p},[\mathbf{p}, \rho(t)]] \\
& -i \frac{\eta_{f}}{2 m \hbar}[\mathbf{q},\{\mathbf{p}, \rho(t)\}] .
\end{aligned}
$$

The full, three-dimensional master equation given by Eq. (S33) can be used for studying full dynamics, light-matter entanglement and photon statistics of the system.

\section{HOMODYNE PHOTODETECTION: A COMPARISON OF THEORY AND EXPERIMENT}

The experimental data in Fig. 2 of the main article was obtained by using a scheme more involved, and of more general applicability, than indicated in the simplified detection model presented in the main article. In the laboratory, the position of the nanoparticle was determined from the optical interference between the unscattered probe and a spherical wave radiated by the induced dipole of the polarizable sphere. The interference signal varies linearly with the particle position for oscillation amplitudes small compared to the optical wavelength. In order to detect the particle position and also to eliminate the large constant background term (equal to the unscattered probe flux $\approx \Phi$ ) in an experimental setting, we leveraged a balanced homodyne detection scheme, as described in [3] and [5].

In this configuration, one of the detectors sampled the entire spatial profile of the probe beam, while the other was apodized so that only the center of the beam was sampled. As the trapped particle moved in the axial direction, the relative amount of scattered light collected by each detector, and thus the AC term of the resulting homodyne current, was modulated. The optical channels were adjusted so that each detector recorded the same average power, and the resulting homodyne signals were subtracted to eliminate the common DC bias.

In our theoretical model, rather than include an additional quantized mode corresponding to the full induced dipole emission pattern, we considered for simplicity only the field scattered back into the probe mode by the nanoparticle. This sim- plification is admissible as long as the value of the linear optomechanical coupling constant $\chi$ in the model is taken from experiment [3]. We emphasize that our experimental method of feedback cooling works even if the trap and probe foci are very close to each other, in which case the optomechanical coupling is essentially quadratic in the particle position, while the position detection (using the dipole wave mode) is still linear. In the theoretical calculation of the phonon number, the linear (or quadratic) optomechanical coupling simply adds a position offset (or frequency shift) to the trap. Both the offset as well as the shift are negligible for weak probe light used in the experiment, rendering the theoretical predictions of ground state cooling identical for linear as well as quadratic optomechanical coupling.

\section{COMPUTING THE GAS DAMPING RATE AS A FUNC- TION OF GAS PRESSURE}

The damping rate of the particle motion due to the background gas is given by $\Gamma_{0} \equiv \eta_{f} / m$, where $m$ is the particle mass and $\eta_{f}=6 \pi \mu r_{d}$ is the coefficient of friction for a spherical particle of radius $r_{d}$ in a fluid of dynamic viscosity $\mu$. This expression is for a particle much larger than the mean free path of the gas bath $\lambda_{\mathrm{mfp}}$. For a nanoparticle in a rarefied gas, as assumed in Eq. (9) in the main text, the damping constant becomes [6]

$$
\begin{aligned}
\Gamma_{0} & =\frac{6 \pi \mu r_{d}}{m} \times \text { correction } \\
& =\frac{6 \pi \mu r_{d}}{m} \frac{0.619}{0.619+\mathrm{Kn}}\left(1+\frac{0.31 \mathrm{Kn}}{0.785+1.152 \mathrm{Kn}+\mathrm{Kn}^{2}}\right),
\end{aligned}
$$

where the correction term is in terms of the Knudsen number $\mathrm{Kn} \equiv \lambda_{\mathrm{mfp}} / r_{d} \propto 1 / P r_{d}$. Using the fact that $\lambda_{\mathrm{mfp}} \approx 70 \mathrm{~nm}$ at atmospheric pressure, and matching the experimentally measured rates $[3,5]$, we can write the damping for a general pressure and particle size as

$$
\Gamma_{0} \approx \frac{r_{d}}{70 \mathrm{~nm}} \frac{2 \pi \times 10^{6} \mathrm{~Hz}}{0.619+\mathrm{Kn}}\left(1+\frac{0.31 \mathrm{Kn}}{0.785+1.152 \mathrm{Kn}+\mathrm{Kn}^{2}}\right)
$$

In order to ensure that this model of damping holds in the deep quantum regime [7], we consider the effects of individual scattering events as described by the master equation [8]

$$
\dot{\rho}=\sum_{V} V \rho V^{\dagger}-\frac{1}{2}\left\{V^{\dagger} V, \rho\right\}
$$

where the Lindblad operators are given by

$$
V \sim \exp \left(-i \frac{\Delta p q}{\hbar}\right)
$$

where $\Delta p$ is the momentum transfered to the nanoparticle during a collision with a gas molecule. Now if the momentum kick is sufficiently small, then we can approximate

$$
\exp (-i \Delta p q / \hbar) \approx 1-i \Delta p q / \hbar,
$$

which ultimately allows us to recover the Brownian contribution, derived in [8], to our master equation presented in the main paper. In order for the approximation of Eq. (S39) to hold, it is necessary that $\Delta p \ell_{\mathrm{coh}} / \hbar<1$, where $\ell_{\text {coh }}$ is the coherence length of the system. Considering the coherence length of the system in its ground state, $\ell_{\mathrm{coh}} \approx \ell_{z}$. Using the parameters listed in the main article for the system at $T=4 \mathrm{~K}$, and 
using the maximum RMS thermal momentum change $\Delta p=$ $2 \sqrt{3 m_{\text {gas }} k_{B} T}$ assuming a gas made of $\mathrm{N}_{2}$ gives

$$
\frac{\Delta p \ell_{z}}{\hbar} \approx 0.6<1
$$

which ensures that our linearized treatment of Brownian motion is valid over all considered regimes. This inequality actually overestimates the magnitude of a typical momentum kick as Hydrogen and Helium comprise the majority of the species of gas molecules in an environment at $4 \mathrm{~K}$, and are much lighter than $\mathrm{N}_{2}$.

\section{CONVERTING A MASTER EQUATION TO A LANGEVIN EQUATION}

Consider a master equation in standard Lindblad form

$$
\dot{\rho}=\mathcal{L}_{0} \rho=\frac{1}{i \hbar}[H, \rho]-\sum_{j} \frac{\gamma_{j}}{2} \mathcal{D}\left[L_{j}\right] \rho,
$$

where $H_{0}$ is a Hamiltonian representing the unitary evolution and $\mathcal{D}\left[L_{j}\right] \rho$ is the standard Lindblad dissipation superoperator for the Lindblad operators $L_{j}$ [9]. Now following the quantumstate diffusion model presented by Gisin and Percival [10], the density matrix can be considered as the expectation over pure states e.g. $\rho \equiv \mathrm{E}[|\psi\rangle\langle\psi|]$, whose dynamics are stochastic in nature. The (stochastic) differential increment for the equation of motion for $|\psi\rangle$ representing the unraveling of the master equation given by the QSD model is $[10,11]$

$$
\begin{aligned}
&|\mathrm{d} \psi\rangle=\frac{1}{i \hbar} H \mathrm{~d} t|\psi\rangle \\
&+\sum_{j}\left[\frac{\gamma_{j}}{2}\left(2\left\langle L_{j}^{\dagger}\right\rangle L_{j}-L_{j}^{\dagger} L_{j}-\left\langle L_{j}^{\dagger}\right\rangle\left\langle L_{j}\right\rangle\right) \mathrm{d} t\right. \\
&\left.+\left(L_{j}-\left\langle L_{j}\right\rangle\right) \sqrt{\gamma_{j}} \mathrm{~d} W_{j}\right]|\psi\rangle
\end{aligned}
$$

where $d W_{j}$ are complex differentials representing Wiener processes defined by $\mathrm{E}\left[\mathrm{d} W_{j}\right]=\mathrm{E}\left[\mathrm{d} W_{j}^{*}\right]=\mathrm{E}\left[\mathrm{d} W_{j} \mathrm{~d} W_{k}\right]=0$, and $\mathrm{d} W_{j}^{*} \mathrm{~d} W_{k}=\delta_{j k} \mathrm{~d} t$. Note, this complex Wiener process is equivalent to $\mathrm{d} W=\left(\mathrm{d} R_{1}+i \mathrm{~d} R_{2}\right) / \sqrt{2}$, where $\mathrm{d} R_{j}$ are real valued Wiener increments. Now such a model is not unique, but represents an open quantum system which is continuously monitored by it's environment [12], and thus provides an appropriate trajectory or unraveling for a system that we will ultimately wish to observe in terms of a PSD or measurement spectrum (i.e. which we will continuously monitor).

Now we are interested in expressing the random forces acting on our system due to interaction with the environment (as expressed by the Lindblad operators $L_{j}$ ). Therefore computing the increment for the momentum $p$ of the system gives

$$
\begin{aligned}
\mathrm{d} p= & \langle\mathrm{d} \psi|p| \psi\rangle+\langle\psi|p| \mathrm{d} \psi\rangle+\langle\mathrm{d} \psi|p| \mathrm{d} \psi\rangle+\mathcal{O}\left(\mathrm{d} t^{2}\right) \\
=- & \frac{1}{i \hbar}\left[H_{0}, p\right] \mathrm{d} t \\
& \quad-\sum_{j} \frac{\gamma_{j}}{2} \mathcal{D}\left[L_{j}^{\dagger}\right] p \mathrm{~d} t+\sum_{j}\left(\mathrm{~d} B_{j}^{\dagger} p+p \mathrm{~d} B_{j}\right) \\
= & \mathcal{L}_{0}^{\#}[p] \mathrm{d} t+\sum_{j}\left(\mathrm{~d} B_{j}^{\dagger} p+p \mathrm{~d} B_{j}\right),
\end{aligned}
$$

where $\mathrm{d} B_{j} \equiv\left(L_{j}-\left\langle L_{j}\right\rangle\right) \sqrt{\gamma_{j}} \mathrm{~d} W_{j}$. Applying this to our master equation gives us our Langevin equation for the stochastic forces listed in Eq. (15) in the main article.

\section{REFERENCES}

1. A. Aiello, C. Marquardt, and G. Leuchs, "Transverse angular momentum of photons," Phys. Rev. A 81, 053838 (2010).

2. C. Gerry and P. Knight, Introductory Quantum Optics (Cambridge University Press, Cambridge, 2004).

3. J. Gieseler, B. Deutsch, R. Quidant, and L. Novotny, "SubKelvin Parametric Feedback Cooling of a Laser-Trapped Nanoparticle," Phys. Rev. Lett. 109, 103603 (2012).

4. H. Wiseman and G. Milburn, "Quantum theory of optical feedback via homodyne detection," Phys. Rev. Lett. 70, 548-551 (1993).

5. L. P. Neukirch and A. N. Vamivakas, "Nanooptomechanics with optically levitated nanoparticles," Contemporary Physics 56, 48-62 (2015).

6. S. A. Beresnev, V. G. Chernyak, and G. A. Fomyagin, "Motion of a spherical particle in a rarefied gas. Part 2. Drag and thermal polarization," Journal of Fluid Mechanics 219, 405 (2006).

7. K. Hornberger, J. E. Sipe, and M. Arndt, "Theory of decoherence in a matter wave Talbot-Lau interferometer," Phys. Rev. A 70, 053608 (2004).

8. L. Diósi, "Quantum Master Equation of a Particle in a Gas Environment," Europhys. Lett. 30, 63-68 (1995).

9. G. Lindblad, "On the generators of quantum dynamical semigroups," Commun. Math. Phys. 48, 119-130 (1976).

10. N. Gisin and I. C. Percival, "The quantum-state diffusion model applied to open systems," J. Phys. A. Math. Gen. 25, 5677-5691 (1992).

11. J. Halliwell and A. Zoupas, "Quantum state diffusion, density matrix diagonalization, and decoherent histories: A model," Phys. Rev. D 52, 7294 (1995).

12. T. A. Brun, "Continuous measurements, quantum trajectories, and decoherent histories," Phys. Rev. A 61, 31 (1997). 